\title{
Effect of Percent Relative Humidity, Moisture Content, and Compression Force on Light-Induced Fluorescence (LIF) Response as a Process Analytical Tool
}

\author{
Ishan G. Shah ${ }^{1,2}$ and William C. Stagner ${ }^{1,3}$
}

Received 22 July 2015; accepted 22 September 2015; published online 9 October 2015

\begin{abstract}
The effect of percent relative humidity (16-84\% RH), moisture content (4.2-6.5\% $w / w \mathrm{MC})$, and compression force (4.9-44.1 $\mathrm{kN} \mathrm{CF}$ ) on the light-induced fluorescence (LIF) response of $10 \% \mathrm{w} / \mathrm{w}$ active pharmaceutical ingredient (API) compacts is reported. The fluorescent response was evaluated using two separate central composite designs of experiments. The effect of \% RH and CF on the LIF signal was highly significant with an adjusted $R^{2}=0.9436$ and $p<0.0001$. Percent relative humidity $(p=0.0022), \mathrm{CF}(p<0.0001)$, and $\% \mathrm{RH}^{2}(p=0.0237)$ were statistically significant factors affecting the LIF response. The effects of MC and CF on LIF response were also statistically significant with a $p$ value $<0.0001$ and adjusted $R^{2}$ value of 0.9874 . The LIF response was highly impacted by MC $(p<0.0001)$, CF $(p<0.0001)$, and $\mathrm{MC}^{2}(p=0022)$. At 10\% w/w API, increased \% RH, MC, and CF led to a nonlinear decrease in LIF response. The derived quadratic model equations explained more than $94 \%$ of the data. Awareness of these effects on LIF response is critical when implementing LIF as a process analytical tool.
\end{abstract}

KEY WORDS: design of experiments (DOE); fluorescence quenching; light-induced fluorescence (LIF); moisture; process analytical technology (PAT).

\section{INTRODUCTION}

Light-induced fluorescence (LIF) has great potential as a process analytical technology (PAT) tool. To date, all LIF research reports and commercial scale GMP PAT sensors used for powders and tablets have been purpose-built. This limits accessibility to solid state PAT LIF technology and its current utility. More than $60 \%$ of the top 200 selling drugs in 2000 had fluorescent structural properties (1). Fluorescence detection has increased sensitivity $(2,3)$, lower detection limits (3), better signal-to-noise ratio (4), and decreased background noise (4) compared to ultraviolet (UV) and near-infrared (NIR) absorption spectroscopy methods. These advantages result from the Stokes shift between the excitation and emission maximum wavelength and the $90^{\circ}$ signal collection angle which minimizes scattered light interference. In addition, fluorescence intensity response is directly proportional to incident irradiance as seen in Eq. 1:

$I_{f}=P_{o} \gamma \phi \& \quad b \quad C$

where $I_{f}$ is the fluorescent intensity, $P_{o}$ the incident irradiance or power per unit area, $\gamma$ the fluorescent collection efficiency,

\footnotetext{
${ }^{1}$ Department of Pharmaceutical Sciences, Campbell University College of Pharmacy \& Health Sciences, Buies Creek, North Carolina, USA.

${ }^{2}$ Data and Clinical Programming Department, PRA Health Sciences, Blue Bell, Pennsylvania, USA.

${ }^{3}$ To whom correspondence should be addressed. (e-mail: bcstagner@gmail.com)
}

$\phi$ the fluorophore quantum efficiency, $\varepsilon$ the molar absorptivity at the fluorophore excitation wavelength, $b$ the optical path length, and $C$ the concentration of the fluorophore (5). The fluorescent intensity equation shows that fluorescent intensity response is directly proportional to the incident irradiance. That is, within limits, increasing the incident power per unit area will lead to increased fluorescence response. This is not the case for UV and NIR absorbance spectroscopic methods. Absorbance is a function of the ratio of $\left(P_{o}\right) /(I)$, where $I$ is the light intensity exiting from the sample and absorbance is $\log$ $\left(P_{o}\right) /(I)$. As a result, the sensitivity of fluorescence is approximately 100-1000 times greater than absorption spectroscopic methods $(4,6)$ which could prove to be an important PAT attribute for low-dose drugs.

As more companies move to continuous processes, PATs that provide inline real-time feedback control become even more critical for manufacturing high-quality cost-effective products. Bakeev (7) has provided a recent compilation of PAT spectroscopic process analysis techniques. Our laboratory has reported the use of inline real-time near-infrared granule moisture content (8) and focused beam reflectance measurement particle size determination (9) for a commercial scale continuous granulation-drying-milling process. Previous studies have shown that LIF can be used in pharmaceutical blending to determine the blending end point $(1,10)$. Karumanchi et al. (10) used off-line LIF to determine blend uniformity, mixing end point, and identification of mixer dead-spots for a commercial scale blending operation. This technique has also shown to be promising in nondestructively measuring online active pharmaceutical ingredient (API) tablet concentration $(11,12)$. Previously, the authors reported the effect of different 
tapped densities, compacted densities, concentration, and the interaction of material density-concentration on LIF response (13). The fluorescent response to powder mixtures containing 0.25 to $10.00 \% w / w$ of the same fluorescent API used in this study was evaluated over a density range of about 0.6 to $1.4 \mathrm{~g}$ / $\mathrm{cm}^{3}$. This density range was achieved by preparing tapped and compressed compacts. Material concentrations up to $4.00 \% \mathrm{w} / \mathrm{w}$ API and evaluated over a 0.6 to $1.4 \mathrm{~g} / \mathrm{cm}^{3}$ material density (MD) range showed a linear LIF response with increasing tapped and compacted density. API concentrations of $4.00 \% \mathrm{w} / w$ to $10 \% \mathrm{w} / \mathrm{w}$ API exhibited a negative parabolic LIF response. These LIF responses were fit to a quadratic model equation that included $\mathrm{MD}, \mathrm{API} \% w / w, \mathrm{MD}^{2},(\mathrm{API} \% w / w)^{2}$, and a MD-API\% $w / w$ interaction term (adjusted $R^{2}=0.975$ and $p$ value $<0.0001$ ). All model terms were highly significant ( $p$ value $<0.0001$ ).

Fluorescence response can be affected by $\mathrm{pH}$, viscosity, metal ions, temperature, and moisture. The fluorescent charge and resonance species can be affected by $\mathrm{pH}$. As kinetic energy increases with increasing temperature, the number of increased fluorophore collisions results in fluorescent quenching by intermolecular energy transfer (14).

Previous studies have shown that the presence of moisture due to water and humidity can lead to fluorescence intensity quenching (15-18). Mishra et al. (17) studied the fluorescence quenching of salicylic acid (SA) and its sodium salt in a polyvinyl alcohol (PVA) film as a function of relative humidity and water/dioxane solution concentration. The authors exposed SA-doped PVA film to different percent relative humidities (\% RH) ranging from 5 to $85 \% \mathrm{RH}$. They observed that the fluorophore response decreased nonlinearly as exposure to \% RH increased. In another experiment, a SA containing dried PVA film was exposed to different water/ dioxane mixtures ( 0 to $60 \% v / v$ water). In this study, there was an initial dramatic linear decrease in fluorescence intensity with exposure to increased amounts of water followed by a nonlinear decrease in fluorescent response to increased amount of water. McGaughey et al. (18) developed a sol-gelbased optical sensor for the measurement of \% RH which was based on the changes in the fluorescent intensity. The \% RH detection limit was $0.35 \% \mathrm{RH}$ indicating that fluorophore response can be sensitive to very small changes in \% $\mathrm{RH}$. Guay et al. (2) have recently reported the development of a multiple fluorophor full emission spectra (302-1149 nm) diode array LIF detector for inline quantitative analysis of pharmaceutical granulations. The effect of moisture, hydrostatic pressure, particle size, and dyes on the LIF signal was evaluated. The study showed that higher moisture content resulted in a decrease in LIF peak for starch, which was attributed to fluorescence signal quenching.

The aim of this study was to determine the effect of \% $\mathrm{RH}$, moisture content (MC), and compression force (CF) on the LIF response. A central composite design (CCD) was employed to quantitate and statistically evaluate the effect of multiple variables, their interactions, and quadratic effects on the LIF response. Experimental approaches controlling one variable at a time are not capable of quantifying and statistically evaluating the significance of multivariable interactions. It was hypothesized that at the concentration studied, \% $\mathrm{RH}$, $\mathrm{MC}$, and $\mathrm{CF}$ main factor effects; \% $\mathrm{RH}-\mathrm{CF}$ and $\mathrm{MC}-\mathrm{CF}$ interaction terms; and \% $\mathrm{RH}, \mathrm{MC}$, and $\mathrm{CF}$ squared effects would have a statistically significant negative impact on the LIF signal. Water is ubiquitous. It is found in pharmaceutical materials such as sorbed water and crystalline water. It is used in granulation processes. Water is present in the atmosphere and \% $\mathrm{RH}$ is often considered a critical manufacturing process variable that is routinely controlled. Environmental moisture can be sorbed by processing powders and cause clumping and aggregation which can lead to poor powder flow (19-21) and content uniform problems (22). Sorbed environmental moisture also can cause moisture sensitive drugs to degrade during the manufacturing process $(23,24)$. Likewise, moisture content resulting from a wet granulation process can affect granule friability (25) and compressibility $(26,27)$, tablet or roller compact hardness and friability (21), and drug stability. Compression force is a process parameter that can be measured during tableting or roller compaction. Over a defined compression range, an increase in compression force will lead to an increase in material density. As noted earlier, the LIF signal is a function of material density and API concentration (13). Therefore, in this particular study, compression force can be considered a measureable process variable that is a surrogate for material density. Knowledge of the effect of \% RH, MC, and compressional force on the LIF response is critically important in developing and employing LIF as a PAT tool.

\section{MATERIALS AND METHODS}

\section{Materials and Instrumentation}

\section{Materials}

A $10.00 \% w / w$ fluorescent API granulation having a particle size of about $110 \mu \mathrm{m}$ was supplied by GlaxoSmithKline, Zebulon, NC. Lactose monohydrate, US National Formulary, modified-spray dried, was used as the diluent. It was purchased from Foremost Farms (Baraboo, Wisconsin). Salts used to prepare the saturated salt solutions at $25 \pm 2^{\circ} \mathrm{C}$ were calcium bromide $\left(\mathrm{CaBr}_{2} \cdot 2 \mathrm{H}_{2} \mathrm{O}\right)$, ACS reagent grade, lot no. WH0428; calcium chloride $\left(\mathrm{CaCl}_{2} \cdot 2 \mathrm{H}_{2} \mathrm{O}\right)$, USP, EP, BP, JP, lot no. V0242; calcium nitrate $\left(\mathrm{Ca}\left(\mathrm{NO}_{3}\right)_{2} \cdot 4 \mathrm{H}_{2} \mathrm{O}\right)$, ACS reagent grade, lot no. ZC1156; sodium chloride $(\mathrm{NaCl})$, ACS reagent grade, lot no. 4X0645; and potassium chloride (KCl) USP, lot no. WP0325 supplied by Spectrum Chemical, New Brunswick, NJ. Karl Fisher reagents were purchased from EMD (Billerica, MA): Combititrant 5, lot no. 46292; Combimethanol, lot no. 48095; and Equastar water standard $1 \%$ v/v, lot no. HC784282.

\section{Purpose-Built LIF Sensor}

A detailed description of the purpose-built LIF sensor including a fiber optic probe has been previously described (13). A photograph of the portable LIF sensor is shown in Fig. 1a, and a general LIF photometric pictorial diagram is illustrated in Fig. 1b. Fluorescent excitation and detection was accomplished using a xenon strobe light source and photomultiplier tube (PMT) style detector. The strobe had an adjustable energy range of $100-180 \mathrm{~mJ}$ at $1-20 \mathrm{~Hz}$. The wavelength range was $190-650 \mathrm{~nm}$ and was configured with excitation and emission filters appropriate for the API. For 

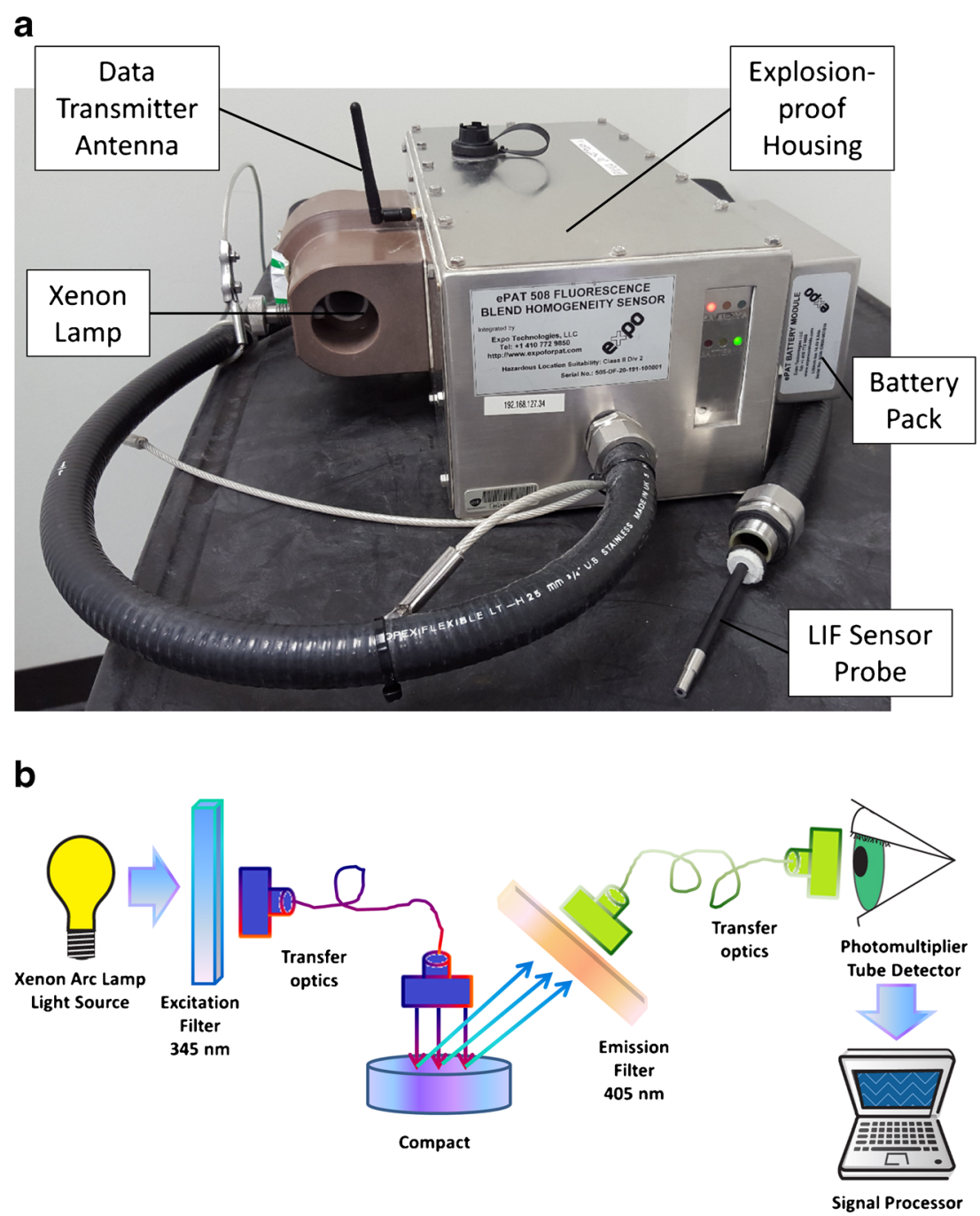

Fig. 1. a Portable LIF sensor. b LIF photometric pictorial diagram

this study, the module unit was fitted with optical filters that provided maximum excitation and emission at 345 and $405 \mathrm{~nm}$, respectively. The PMT had an adjustable gain ranging from 26 to 52 . The sensor offset was $250 \mathrm{~ns}$ at a strobe period of $100 \mathrm{~ms}$.

\section{Karl Fisher Water Titrator}

An Aquastar ${ }^{\circledR}$ Karl Fischer water titrator, model no. AQU33 was used to make water measurements of granulations stored at various relative humidity conditions.

\section{Methods}

\section{Calibration of LIF Sensor}

The LIF sensor calibration was performed using reference standards (Spectralon ${ }^{\circledR}$ Labsphere, Inc. Sutton, NH) and placing the LIF fiber optic probe directly on the reference standard glass plate. The LIF PMT responses were plotted against the reference standard values and the coefficient of determination $\left(R^{2}\right)$ was calculated. According to the fluorescence standard supplier, the sensor was adequately calibrated if the $R^{2}$ was 0.950 or greater. To maximize the signal-to-noise ratio and achieve the largest $R^{2}$ value, a sensitivity of 43 was chosen for measuring the LIF response of the compacts.

\section{Central Composite Experimental Design and Data Analysis}

A central composite experimental design with five center points (JMP Pro 10 Statistical Software: SAS, Cary, NC) was chosen to study the effect of \% RH, MC, and CF on the LIF response. The central composite design supports the quantitation and statistical evaluation of the LIF response as a function of multiple independent factors or variables including their interaction terms and quadratic effects. Equation 2 is a representative model equation using \% $\mathrm{RH}$ and $\mathrm{CF}$ as the independent variables:

$$
\begin{aligned}
\text { LIF response }= & a_{0}+a_{1}(\% \mathrm{RH})+a_{2} \mathrm{CF} \\
& +a_{12}(\% \mathrm{RH}) \mathrm{CF}(\text { the interaction term }) \\
& +a_{11}(\% \mathrm{RH})^{2}+a_{22} \mathrm{CF}^{2}+\varepsilon
\end{aligned}
$$


where $a_{0}$ is the intercept which is the arithmetic mean when no model effects are specified, $a_{1}$ and $a_{2}$ are the coefficients for the main effects, $a_{12}$ is the coefficient of the interaction term, $a_{11}$ and $a_{22}$ are the coefficients of each variable's quadratic effect, and $\varepsilon$ is the experimental variability. The five center points were chosen to improve the estimate of the study reproducibility and precision. The center points also aid in modeling potential quadratic effects. Interactions between variables are observed that when the level of one variable is changed, the effect of the other variable also changes. Quadratic or squared effects can lead to a curvilinear response surface that exhibits a maximum or minimum in the observed response. The design of experiments (DOE) yielded 13 randomized runs with 5 center points and are shown in Tables I and II. The influence of \% RH and MC was evaluated in two separate, but identical, designs. Analysis of variance and development of predictive model equations using least squares of effects were performed for both DOE studies.

\section{API Exposure to Controlled Relative Humidity}

The API granules were stored in air-tight glass desiccator jars containing saturated salt solutions. The desiccators were placed in a validated environmental chamber (Environmental Specialties, Inc. Raleigh, NC) that was maintained at $25 \pm 2^{\circ} \mathrm{C}$. Table III lists the five salts used and their saturated relative humidities which ranged from 16.5 to $84.3 \% \mathrm{RH}$. Temperature-humidity loggers (Sper Scientific, Scottsdale, AZ) were used to confirm each desiccator's \% RH. A thin layer of API was weighed in an inert weigh boat to a tenth of a milligram and placed in one of the equilibrated humidity chambers. Each sample was weighed at regular intervals until an equilibrium weight was achieved. The weight change of the samples was monitored. All samples reached equilibrium within 7-15 days.

Table I. Effect of Percent Relative Humidity (\% RH) and Compression Force on Light-Induced Fluorescence (LIF) Response Using a Central Composite Experimental Design with Five Center Points

\begin{tabular}{lccc}
\hline Pattern $^{a}$ & \% RH & Compression force, kN & Mean LIF response \\
\hline-+ & 30.0 & 39.2 & 66.0 \\
00 & 51.0 & 24.5 & 64.0 \\
A0 & 84.3 & 24.5 & 59.9 \\
+- & 75.3 & 9.8 & 65.8 \\
00 & 51.0 & 24.5 & 63.9 \\
$0 \mathrm{~A}$ & 51.0 & 44.1 & 59.8 \\
00 & 51.0 & 24.5 & 64.0 \\
++ & 75.3 & 39.2 & 59.6 \\
00 & 51.0 & 24.5 & 64.1 \\
00 & 51.0 & 24.5 & 64.2 \\
a0 & 16.5 & 24.5 & 65.0 \\
-- & 30.0 & 9.8 & 67.3 \\
$0 \mathrm{a}$ & 51.0 & 4.9 & 69.9 \\
\hline
\end{tabular}

$a$ " 00 " shows a center point, any combination showing "a" indicates the lowest value and " $\mathrm{A}$ " indicates the highest value of \% $\mathrm{RH}$ or compression force whereas "+" is the upper and "-" is the lower limit of the factors
Table II. Effect of Moisture Content and Compression Force on Light-Induced Fluorescence (LIF) Response Using a Central Composite Experimental Design with Five Center Points

\begin{tabular}{llll}
\hline Pattern $^{a}$ & $\begin{array}{c}\text { Moisture content, } \\
\% \text { w } w\end{array}$ & $\begin{array}{c}\text { Compression } \\
\text { force, } \mathrm{kN}\end{array}$ & $\begin{array}{c}\text { Mean LIF } \\
\text { response }\end{array}$ \\
\hline-+ & 4.51 & 39.2 & 61.0 \\
00 & 4.88 & 24.5 & 64.0 \\
$\mathrm{~A} 0$ & 6.53 & 24.5 & 59.9 \\
+- & 5.89 & 9.8 & 65.8 \\
00 & 4.88 & 24.5 & 63.9 \\
$0 \mathrm{~A}$ & 4.88 & 44.1 & 59.8 \\
00 & 4.88 & 24.5 & 64.0 \\
++ & 5.89 & 39.2 & 59.6 \\
00 & 4.89 & 24.5 & 64.1 \\
00 & 4.88 & 24.5 & 64.2 \\
a0 & 4.16 & 24.5 & 65.0 \\
-- & 4.51 & 9.8 & 67.3 \\
$0 \mathrm{a}$ & 4.88 & 4.9 & 69.9 \\
\hline
\end{tabular}

$a$ " 00 " shows a center point, any combination showing "a" indicates the lowest value and " $\mathrm{A}$ " indicates the highest value of moisture content or compression force where as "+" is the upper and "-" is the lower limit of the factors

\section{Karl Fischer Moisture Content Determination}

After all the samples reached an equilibrium weight, the samples were analyzed for moisture content using the method outlined in the Karl Fischer (KF) manual. One percent $v / v$ water standard was used to calibrate the KF instrument. The API granule sample size was about 0.75 to $1 \mathrm{~g}$. Sample weighing and transfer to the KF test solution was done quickly to minimize any change in moisture content. The KF samples were analyzed in triplicate. The water content percent relative standard deviation (\% RSD) range for the five different \% RH samples was 0.576 to 2.15 . The mean \% RSD was 1.40 . This indicates that sample preparation and analysis was well controlled with low variability.

\section{Preparation of Compacts}

Three hundred ninety milligrams of API granulation was accurately weighed and transferred to an 11.2-mm diameter die. The samples were compressed using a Carver® bench-top hydraulic press (Carver Inc. Wabash, IN) to make compacts at five preset compressional forces $(4.9$ to $44.1 \mathrm{kN})$ in the random order designated by the experimental design. Postcompression, the

Table III. Percent Relative Humidity of Saturated Salt Solutions Stored at $25 \pm 2^{\circ} \mathrm{C}$ in Air-Tight Glass Desiccators

\begin{tabular}{lc}
\hline Salt & $\begin{array}{l}\text { Saturated salt solution } \\
\text { Percent relative humidity }\end{array}$ \\
\hline $\mathrm{CaBr}_{2} \cdot 2 \mathrm{H}_{2} \mathrm{O}$ & $16.5(28)^{\mathrm{a}}$ \\
$\mathrm{CaCl}_{2} \cdot 2 \mathrm{H}_{2} \mathrm{O}$ & $30.0(29)$ \\
$\mathrm{Ca}\left(\mathrm{NO}_{3}\right)_{2} \cdot 4 \mathrm{H}_{2} \mathrm{O}$ & $51.0(30)$ \\
$\mathrm{NaCl}$ & $75.3(28)$ \\
$\mathrm{KCl}$ & $84.3(28)$ \\
\hline
\end{tabular}

${ }^{a}$ Reference number 
upper punch was immediately removed and 10 to $20 \mathrm{~s}$ was allowed for elastic recovery. LIF measurements were taken by placing the fiber optic probe directly on the compact and determining the response from three designated locations on the top and bottom of the compact. Triplicate measurements were made at each location. The 18 real-time LIF measurements indicated that there was no measureable change in the moisture content during the data collection process.

\section{RESULTS AND DISCUSSION}

The effect of \% RH and moisture content was evaluated in separate DOE studies. It is not uncommon for \% $\mathrm{RH}$ and $\mathrm{MC}$ to be used as separate moisture control strategies. Percent relative humidity is often a critical manufacturing parameter for compaction processes such as tableting and roller compac- tion. Powder flow and compatibility can be affected by exposure to varying \% $\mathrm{RH}$. Likewise, moisture content is frequently a critical material quality attribute for running powders that will undergo roller compaction or tableting. Moisture content can affect granule and tablet hardness and friability. Drug stability is also often affected by granule and final product moisture content.

The \% RH range studied was 16.5 to $84.3 \% \mathrm{RH}$. The compression force ranged from 4.9 to 44.1 which was similar to the range used in the previous reported study (13). The predictive equation describing the effect of \% RH and CF on the LIF signal was statistically significant with an adjusted $R^{2}=0.9436$ and $p<0.0001$. Percent relative humidity $(p=0.0022)$, compaction force $(p<0.0001)$, and \% $\mathrm{RH}^{2}(p=0.0237)$ were statistically significant factors affecting the LIF response. Equation 3 is the predictive LIF response equation:

LIF response $=64.0-1.8[(\% \mathrm{RH}-50.4) / 33.9]-4.6[(\mathrm{CF}, \mathrm{kN}-24.5) / 19.6]-1.6[(\% \mathrm{RH}-50.4) / 33.9]^{2}$

The model accuracy and statistical significance was improved by taking out statistically insignificant variables from Eq. 2. The model accuracy was evaluated with regards to improvement in $R^{2}$ values and the statistical significance was assessed by a decrease in the model's $p$ value. The scaled parameter estimates shown here relate the magnitude of the effect when the factors are scaled to a mean of 0 and a range of 2. In this particular study design, $\mathrm{CF}$ has about a 2.5 times greater impact on LIF signal than \% RH. The negative coefficients of the main factors indicate that both \% $\mathrm{RH}$ and $\mathrm{CF}$ have a negative effect on the LIF response which was hypothesized. The ability of moisture to cause fluorescence quenching has been reported (15-18). In our previous work (13), material concentrations up to $4.00 \% \mathrm{w} / \mathrm{w}$ of the same API used in this study were evaluated over material density range of 0.6 to $1.4 \mathrm{~g} / \mathrm{cm}^{3}$. The LIF response exhibited a linear increase with increasing tapped and compacted density. The larger densities were achieved using the same Carver Press and compression pressures ranging from about 2 to $44 \mathrm{kN}$. It was speculated that the increase in LIF signal resulted from an increased amount of API per unit volume. This increase in concentration due to increased density (also associated with increase CF) was referred to as the "apparent concentration" compared to the initial blended percent $w / w$ API. API concentrations of $4.00 \% w / w$ to $10 \% w / w$ API exhibited a negative parabolic LIF response over the same density range. At higher material densities, which were generated by forming compacts on a Carver Press, the LIF signal decreased with increasing density. This quenching phenomenon has been ascribed to competing excited state processes, interacting species, and sample matrix effects that can lead to a decrease in LIF response (5). A statistically significant MD-API\% $w / w$ interaction was also observed in this earlier study. This means that when the level of one factor is changed, the effect of the other factor also changes. In the current study, the interaction between \% RH and CF is not statistically significant as hypothesized, and the decrease in LIF response with increasing \%
$\mathrm{RH}$ and CF (also associated material density increase) occurs in an independent fashion versus synergistically. This is an interesting finding requiring further investigation. The lack of a statistically significant interaction between \% RH and CF may be due to the narrow moisture content range investigated in this study. The hypothesized quadratic CF effect was also not statistically significant. It is most likely that the CF forces studied were too high to show a significant quadratic effect. Figure 2 shows the LIF response contour plot of CF versus \% RH whose design space covers a fairly wide range of compressional forces and \% RH. The plot shows a decrease in LIF response with increase $\mathrm{CF}$ and \% RH. A maximum in the LIF response due to the \% RH quadratic effect occurs at around $32-33 \% \mathrm{RH}$.

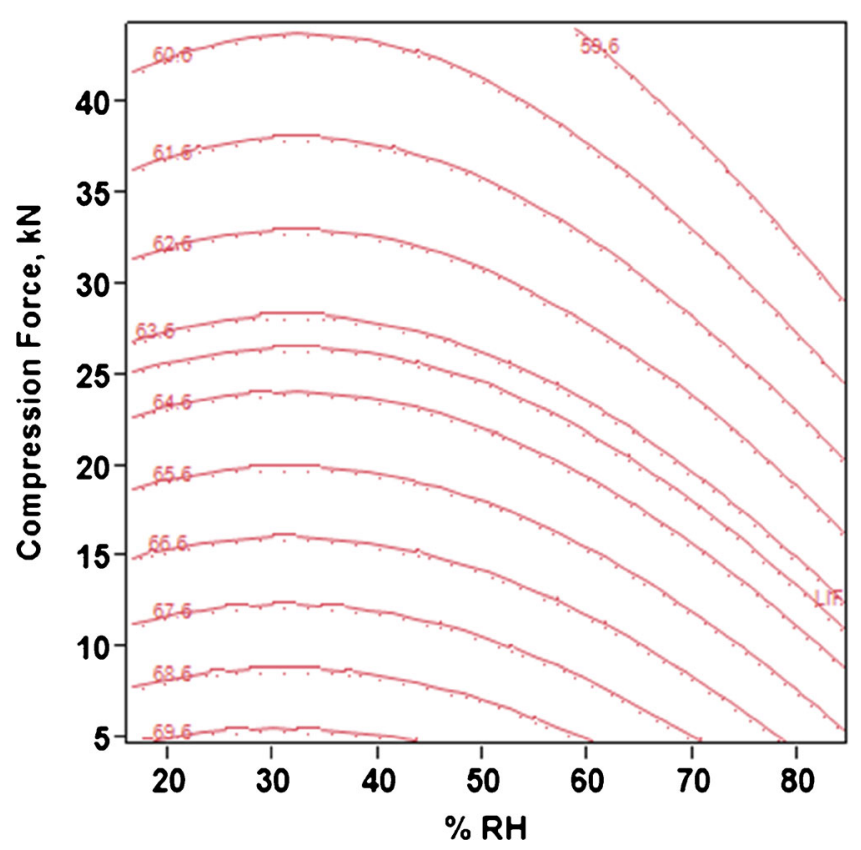

Fig. 2. Light-induced fluorescence (LIF) contour plot as a function of compression force and percent relative humidity (\%RH) 
A relatively narrow API granule moisture content range of 4.16 to $6.53 \% w / w$ water was studied. Analysis of variance showed that $\mathrm{MC}$ and $\mathrm{CF}$ had a statistically significant negative effect on the LIF response $(p<0.0001)$. This shows that the LIF response is sensitive to small changes in the moisture content in powder matrices such as tablet granulations and powder blends. The model equation relating $\mathrm{MC}$ and $\mathrm{CF}$ to the $\mathrm{LIF}$ signal is (Eq. 3):

LIF Response $=69.1-1.9[(\mathrm{MC}, \% w / w-5.3) / 1.2]-4.4[(\mathrm{CF}, \mathrm{kN}-24.5) / 19.6]-1.5[(\mathrm{MC}, \% w / w-5.3) / 1.2]^{2}$

The overall model had an adjusted $R^{2}$ value of 0.9874 . $\mathrm{MC}(p<0.0001), \mathrm{CF}(p<0.0001)$, and $\mathrm{MC}^{2}(p=0.0022)$ exhibited a statistically significant negative impact on the LIF signal. The hypothesized CF squared term's effect approached statistical significance with a $p$ value of 0.1038 . Evaluation of the scaled estimates shows that CF has about a 2.3-fold greater impact on the LIF signal than MC. The interaction term between MC and CF was not statistically significant. Figure 3 is an LIF contour plot for CF versus MC. Figure 3 shows that both MC and CF have a negative effect on LIF response. In addition, the plot shows that quadratic effect becomes more noticeable at around 4.5 to $5 \% \mathrm{w} / \mathrm{w} \mathrm{MC}$.

Considering the present research and our previous work, it might be speculated that at API concentrations of $2.5 \% \mathrm{w} / \mathrm{w}$ and less, an antagonist interaction between $\mathrm{CF}$ and \% $\mathrm{RH}$ or $\mathrm{MC}$ would occur. At these lower API concentrations, increases in CF would be expected to result in a linear increase in LIF response due to an increase in apparent density. On the other hand, an increase in \% RH or MC would be expected to decrease the LIF signal due to quenching. Other investigators $(17,18)$ have shown fluorescence quenching to occur over a very wide \% RH range as well. Therefore, it is likely that that the opposing effects of $\mathrm{CF}$ and \% $\mathrm{RH}$ or MC would result in an antagonist interaction. Further experiments are required to study this phenomenon more fully.

This work has added significant knowledge to the use of LIF in pharmaceutical powder processing. To implement LIF in commercial production, the effect of API concentration

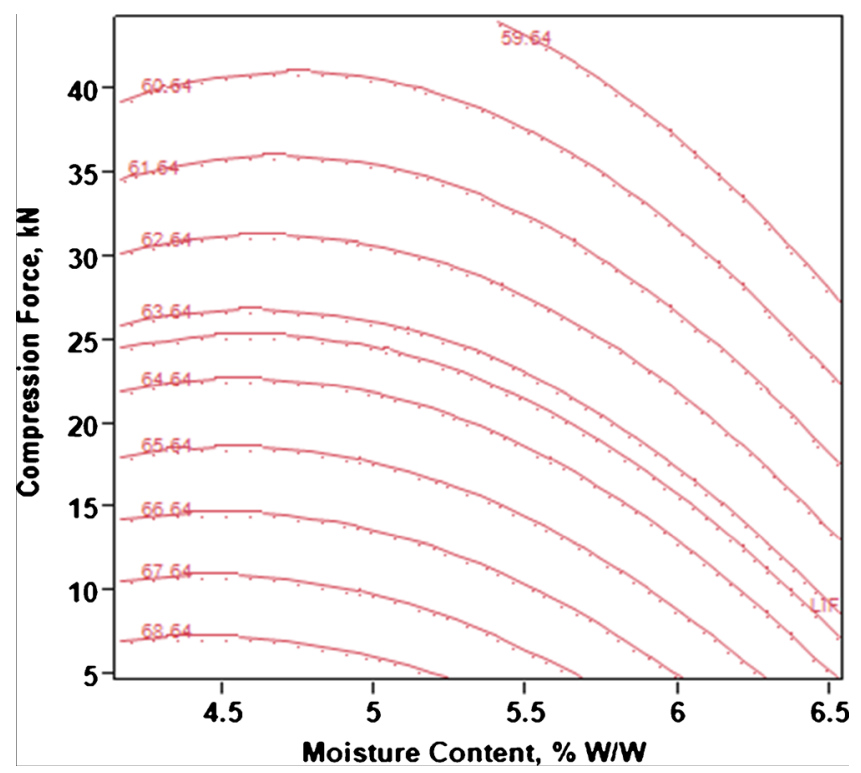

Fig. 3. Light-induced (LIF) contour plot as a function of compression force and moisture content
(13) environmental \% RH, powder moisture content, and $\mathrm{CF}$ will need to be taken into account when developing unique standard curves. With this work, it should be possible to use LIF effectively to track powder concentrations, blend endpoints, and make other PAT quantitative measurements during roller compaction and tableting.

\section{CONCLUSIONS}

The effect of \% RH, MC, and $\mathrm{CF}$ on the LIF response has been reported. LIF response is sensitive to small changes in $\% \mathrm{RH}, \mathrm{MC}$, and CF. At $10 \% w / w$ API, increases in \% RH, $\mathrm{MC}$, and $\mathrm{CF}$ resulted in a nonlinear decrease LIF response. Separate model equations were developed that related \% $\mathrm{RH}$ and $\mathrm{CF}$, and $\mathrm{MC}$ and $\mathrm{CF}$ to the LIF response. These separate models were developed since $\% \mathrm{RH}$ is often considered a critical manufacturing process variable that is routinely controlled while moisture content is often employed as a critical material quality attribute for intermediates such as granulated powders and as a final product attribute test. The models were statistically significant with $p$ values $<0.0001$ and greater than $94 \%$ of the data was fit by the model equations. The interaction terms in the model equations were not statistically significant indicating that the main effects caused the observed decreases in the LIF signal in an independent fashion. Both \% $\mathrm{RH}$ and MC quadratic terms were statistically significant in their respective models. Evaluation of the scaled parameter estimates showed that $\mathrm{CF}$ had a greater than twofold impact on the LIF response compared to \% $\mathrm{RH}$ or MC. The effects of \% RH, MC, and CF need to be considered when developing and implementing LIF PATs for processes such as blending, wet granulation and drying, roller compaction, and tableting.

\section{ACKNOWLEDGMENTS}

The authors would like to thank GlaxoSmithKline for providing the Honeywell H-LIF instrument and the fluorescent API granulation.

\section{REFERENCES}

1. Lai C-K, Cooney CL. Application of a fluorescence sensor for miniscale on-line monitoring of powder mixing kinetics. J Pharm Sci. 2004;93:60-70.

2. Guay J-M, Lapointe-Garant P-P, Gosselin R, Simard J-S, Abatzoglou N. Development of a multivariate light-induced fluorescence (LIF) PAT tool for in-line quantitative analysis of pharmaceutical granules in a V-blender. Eur J Pharm Biopharm. 2014;86:524-31. 
3. Harris DC. Quantitative chemical analysis. 8th ed. New York: W. H. Freeman and Company; 2010. p. 612.

4. Harris DC. Quantitative chemical analysis. 9th ed. New York: W. H. Freeman and Company; 2016. p. 432-60.

5. Dickens JE. Fluorescent sensing and process analytical applications. In: Bakeev K, editor. Process analytical technology. 2nd ed. Chichester: John Wiley \& Sons; 2010. p. 343.

6. Harris DC. Quantitative chemical analysis. 9th ed. New York: W. H. Freeman and Company; 2016. p. 687.

7. Bakeev KA. Process analytical technology. 2nd ed. Chichester: John Wiley \& Sons; 2010.

8. Chablani L, Taylor MK, Mehrotra A, Rameas P, Stagner WC. Inline real-time near-infrared granule moisture measurements of a continuous granulation-drying-milling process. AAPS PharmSciTech. 2011;12:1050-5.

9. Kumar V, Taylor MK, Mehrota A, Stagner WC. Real-time particle size analysis using focused beam reflectance measurement as a process analytical tool for a continuous granulation-dryingmilling process. AAPS PharmSciTech. 2013;14:523-30.

10. Karumanchi V, Taylor MK, Ely KJ, Stagner WC. Monitoring powder blend homogeneity using light-induced fluorescence. AAPS PharmSciTech. 2011;12:1031-7.

11. Lai C-K, Zahari A, Miller B, Katstra WE, Cima MJ, Cooney CL. Nondestructive and on-line monitoring of tablets using light-induced fluorescence technology. AAPS PharmSciTech. 2004;5:1-10.

12. Domike R, Ngai S, Cooney CL. Light induced fluorescence for predicting API content in tablets: sampling and error. Int $\mathbf{J}$ Pharm. 2010;391:13-20.

13. Shah IG, Ely KJ, Stagner WC. Effect of tapped density, compacted density, and drug concentration of light-induced fluorescence (LIF) response. J Pharm Sci. 2015;104:1732-40.

14. Olsen ED. Modern optical methods of analysis. New York: McGraw-Hill; 1975. p. 389-91.

15. Posch HE, Wolfbeis OS. Optical sensors, 13: fibre-optic humidity sensor based on fluorescence quenching. Sensors Actuators. 1988;15:77-83.

16. Otsuki S, Adachi K. Effect of humidity on the fluorescence properties of a medium-sensitive fluorophore in a hydrophilic polymer film. J Photochem Photobiol A Chem. 1993;71:169-73.

17. Mishra H, Misra V, Mehata MS, Pant TC, Tripathi HB. Fluorescence studies of salicylic acid doped poly(vinyl alcohol) film as a water/humidity sensor. J Phys Chem A. 2004;108:2346-52.

18. McGaughey O, Ros-Lis JV, Guckian A, McEvoy AK, McDonagh C, MacCraith BD. Development of a fluorescence lifetime-based sol-gel humidity sensor. Anal Chim Acta. 2006;570:15-20.

19. Emery E, Oliver J, Pugsley T, Sharma J, Zhou J. Flowability of moist pharmaceutical powders. Powder Technol. 2009;189:409-15.

20. Sandler N, Reiche K, Heinämäki J, Yliruusi J. Effect of moisture on powder flow properties of theophylline. Pharmaceutics. 2010;2:275-90.

21. Nokhodchi A. An overview of the effect of moisture on compaction and compression. Pharm Technol. 2005:46-66.

22. Singhai SK, Chopra VS, Nagar M, Jain N, Trivedi P. Scale up determination of $\mathrm{V}$ blender: an overview. Pharm Lett. 2010;2:408-33.

23. Ahlneck C, Zografi G. The molecular basis of moisture effects on the physical and chemical stability of drugs in the solid state. Int $\mathrm{J}$ Pharm. 1990;62:87-95.

24. Kamada K, Yoshimura S, Murata M, Murata H, Nagai H, Ushio $\mathrm{H}$, et al. Characterization and monitoring of pseudopolymorphs in manufacturing process by NIR. Int $\mathrm{J}$ Pharm. 2009;368:103-8.

25. Watano S, Takashima H, Miyanami K. Scale-up of agitation fluidized bed granulation. V. Effect of moisture content on scale-up characteristics. Chem Pharm Bull. 1997;45:710-4.

26. Wade JB, Martin GP, Long DF. A methodological approach for determining the effect of moisture content on the compaction properties of powders: granular hydroxyapatite. Powder Technol. 2013;246:511-9.

27. Chowhan ZT, Chow YP. Compression properties of granulations made with binders containing different moisture contents. J Pharm Sci. 1981;70:1134-9.

28. Greenspan L. Humidity fixed points of binary saturated aqueous solutions. J Res Natl Bur Stand-A Phys Chem. 1977;81:89-96.

29. Stokes RH, Robinson RA. Standard solutions for humidity control at $25^{\circ}$ C. Ind Eng Chem. 1949;41:2013.

30. Quantifoil instruments. www.qinstruments.com/uploads/media/ humidity.pdf. Accessed 28 May 2015. 